\title{
An interdisciplinary and collaborative initiative in palliative care research
}

\author{
Veena Desa' \\ Cyril Danjoux ${ }^{2}$ \\ Yvette Matyas' \\ Margaret Fitch ${ }^{3}$ \\ Amna Husain ${ }^{4}$ \\ Nina Horvath ${ }^{5}$ \\ Jeff Myers ${ }^{5}$ \\ Mark Clemons ${ }^{6}$ \\ Janet $\mathrm{E} \mathrm{Hux}$, 7,8 \\ Elizabeth A Barnes ${ }^{2}$ \\ 'Department of Administration; \\ ${ }^{2}$ Department of Radiation Oncology; \\ ${ }^{3}$ Psychosocial and Behavioral Research \\ Unit, Toronto Sunnybrook Regional \\ Cancer Centre; ${ }^{4}$ The Temmy Latner \\ Centre for Palliative Care, Mount Sinai \\ Hospital, Toronto, Ontario, Canada; \\ ${ }^{5}$ Department of Family Medicine, \\ Sunnybrook Hospital, Toronto, \\ Ontario, Canada; ${ }^{6}$ Department of \\ Medical Oncology, Toronto, Ontario, \\ Canada; ${ }^{7}$ Institute for Clinical \\ Evaluative Sciences, Toronto, Ontario, \\ Canada; ${ }^{8}$ Department of Health \\ Policy, Management, and Evaluation, \\ University of Toronto, Toronto, \\ Ontario, Canada
}

Correspondence: Elizabeth A Barnes Department of Radiation Oncology, Toronto Sunnybrook Regional Cancer Centre, 2075 Bayview Ave, Toronto,

Ontario, Canada M4N 3M5

Tel + I 4l 6480495 |

$\mathrm{Fax}+$ I 4164806002

Email toni.barnes@sunnybrook.on.ca

\begin{abstract}
The scale and complexity of palliative care increasingly demands that researchers move beyond their own discipline and explore interdisciplinary collaboration. At a Palliative Care Research Retreat held in January 2006 at the Toronto Sunnybrook Regional Cancer Centre, researchers from multiple care settings with the center and from other Toronto hospitals came together with the vision of becoming Canadian leaders in palliative care research. As a result of this retreat, five interdisciplinary groups were formed to pursue research in the areas of pain and symptom management, access to services, translational research, education, and communication. An overview of the retreat and direction of research for each group is provided.
\end{abstract}

Keywords: palliative care, research retreat, pain and symptom management, access to care, translational research, palliative care education, communication

\section{Introduction}

The number of deaths in Canada is expected to increase $33 \%$ by the year 2020 and the current number of Canadians with access to hospice palliative care is estimated to be $15 \% .{ }^{1}$ Each death potentially affects the immediate well being of an average of five other people, or more than one million Canadians each year. ${ }^{2}$

The most promising approach to improving the quality of palliative care is the generation of new knowledge through research. Rigorous scientific research can provide the evidence for informed decision-making by clinical practitioners and policy makers. ${ }^{3}$ To be useful in providing new insights, such research should involve collaboration across disciplines and encourage researchers to develop innovative approaches. In order for such collaboration to occur, there must be infrastructure in place to support this. On January 13, 2006, the Rapid Response Radiotherapy Program (RRRP) in collaboration with the Toronto Sunnybrook Palliative Care Initiative, the Psychosocial and Behavioral Research Unit, Bone Metastases Clinic, and the Temmy Latner Centre for Palliative Care at Mount Sinai Hospital sponsored a palliative care research retreat. The vision of this retreat was to become a Canadian leader in interdisciplinary palliative care research.

Objectives of this retreat included:

- To have a shared overview of palliative care research,

- To learn about each other's research and research interests,

- To identify clinical synergies that might foster research questions,

- To identify common research areas and questions,

- To identify strategies to increase research collaboration.

In order to facilitate the widest possible overview of palliative care research, the retreat brought together 32 participants representing the following professions: physicians (oncologists, palliative care physicians, surgeons), nurses, pharmacists, social workers, psychologists, basic scientists, radiation therapists, and administrators. 
Prior to the retreat, attendees were asked to submit their research publications for the past two years, as well as their current research interests. An extraction of themes from the submitted publications showed that there were ten areas of research concentration: symptom assessment, patients' perspective and experience, communication, workforce issues, program evaluation, technology, health services, knowledge transfer-exchange, intervention, and research expertise.

\section{Emerging areas of interdisciplinary research}

From the ten research themes identified, participants were asked to select three themes that were of interest to them. The five most popular choices for interdisciplinary research were pain and symptom management, access to palliative care services, translational research, education, and communication. Each group was charged with formulating a research question, brainstorming strategies, and discussing next steps to investigate their research question. The following sections will discuss the overview and direction of research for each group.

\section{Pain and symptom management}

Research in the area of symptom clusters is likely to make a significant contribution to the state of scientific knowledge in this nascent area and is likely to have a broad impact on patients through the progression of their condition. Although a definition of a symptom cluster has been proposed by Dodd and colleagues, the definition has yet to be tested and conceptual understanding of symptom clustering has to be developed. ${ }^{4}$

Some early work has identified fatigue, sleep disturbance, and pain as a cluster in cancer patients. ${ }^{5}$ The evidence for the prevalence and significance of this cluster and the identification of other clusters remains in large part to be studied.

Understanding symptom clusters will allow the design of interventions and outcome measures in trials that more closely reflect patients' experiences. Improving the conceptual understanding of symptom clustering will provide the evidence-based knowledge to the experiential knowledge of the interaction and impact of multiple symptoms in patients. The evidence-based knowledge will improve the efficiency and efficacy of dissemination of this clinical experience to new generations of health care providers. The direct clinical utility of symptom cluster work is that it will inform the timing and choice of therapeutic strategies when faced with patients with multiple symptoms. Symptom cluster work will make it possible to explore the complex set of clinical questions that clinicians face when caring for patients.

\section{Access to palliative care services}

Access is a complex construct, and may be influenced by health system factors (provider training, provider reimbursement, integration of care), provider factors (knowledge and attitude toward end of life care, knowledge regarding local services) and patient factors (knowledge and attitudes toward palliative care, language and culture barriers).

Utilization is more easily measured but may reflect an even more complex interplay of service availability and accessibility. A clinical concern is that palliative services are under-utilized by patients with identified end-stage illness (both cancer and other diseases) and that the proportion of patients not receiving appropriate care may be greater outside of an oncology context.

Two approaches will be used to assess access to palliative care services in Ontario. The first is to use health care administrative data to examine the extent of the problem of under-use of palliative care services among cancer patients. The second is to locally examine some of the patient and physician level barriers to accessing palliative care at Sunnybrook. A study will aim to determine whether implementation of an end of life care pathway can improve the rate of referral to palliative care by general internal medicine and improve the end of life care provided by a medicine unit.

\section{Translational research}

One of the fundamental problems of performing true translational research in palliative care revolves around the actual meaning of "translation". Many millions of dollars are spent in more traditional aspects of translational research. Indeed, many clinical trials in metastatic cancer (a situation in which most patients are palliative) have translational components. For example, there has been a rapid expansion in studies of novel targeted therapies looking at tumor characteristics that might predict for clinical benefit. Pivotal research took the observation that women with HER2-amplified breast cancer had a poorer prognosis through to the development of the recombinant humanized monoclonal antibody, trastuzumab (herceptin), directed against HER2 for the treatment of HER2-positive breast cancer. This agent has shown remarkable results in the metastatic setting and is now a standard of care for women with early breast cancer that over-expresses HER2. ${ }^{6}$

Can such gains be obtained in other translational settings in palliative care? It is clear that despite the stunning advances 
in the management of bone metastases in recent years that most patients continue to suffer the morbidity and mortality consequences of these metastases. This clinical observation can be truly translated back to the bench. By analyzing tumor specimens from bone metastases, the in vivo mechanisms of bone destruction can be studied despite modern anticancer therapy. If the mechanism of bone breakdown is elucidated then it will allow the development of rational new strategies to improve patient management. In addition, as bisphosphonate therapy works through their osteoclast-inhibiting effects, the occurrence of bone destruction in breast cancer patients occurs in the near absence of osteoclasts. This phenomenon has been reported in previous studies of biopsies from bone metastases and it raises important questions about the continued use of bisphosphonates until death that is often seen in metastatic breast cancer patients.

\section{Education}

The knowledge base in palliative and end of life care is continuing to rapidly expand. There is a need for those with an interest in education and palliative medicine to have effective means and opportunities to transfer their knowledge to undergraduate and postgraduate students. Currently there exists a dearth of information as to how to best provide an adequate educational experience at all levels of training.

Models of innovative educational experiences will be developed with the intent to evaluate the success of their implementation. For example, the College of Family Physicians of Canada is poised to adopt a set of competencies in palliative medicine and it is expected that by 2007 each program will need to demonstrate adequate curricular integration in order to achieve accreditation. It is currently unclear how best to integrate information into an already saturated program. By working collaboratively with the Family Medicine Program, unique models of curricular integration will be developed and evaluated.

\section{Communication}

Communication is an important element in palliative and supportive care. The quality of communication with patients, family, and team members has been found to be fundamental to the quality of care for the patients at the end of life. ${ }^{7}$ The need to provide opportunities for health care providers to improve their communication skills has been recognized. ${ }^{8}$ Poor communication can leave clinicians and family members stressed and dissatisfied, as well as patients' wishes neglected.
Many issues need to be addressed in communication research. For example, when providing palliative and supportive care services, questions arise as to how information should be given, especially in the electronic age. Other issues include determining the optimal process and content, communicating with marginalized groups, and recognizing diversity in cultural and ethnic backgrounds, with the latter being especially relevant in Toronto.

\section{Conclusion}

The January 2006 Palliative Care Research Retreat was successful in bringing together people from different disciplines and with different areas of expertise, all with a common interest in palliative care research. By integrating different viewpoints and fostering communication, such collaboration can help to build an environment that facilitates palliative care research. Following the positive response to the first palliative care research retreat, we are now planning to hold this retreat on an annual basis. Our goal of promoting collaborative palliative care research will be measured in part by the generation of projects, success in grant applications, and publications in peer reviewed journals. Ultimately, the aim is to improve the access and quality of palliative care for patients at the end of life and their families.

\section{Acknowledgments}

We would like to thank all those who attended the palliative research retreat. The authors report no conflicts of interest in this work.

\section{References}

1. Quality End of Life Care Coalition of Canada. Framework for a national strategy on palliative and end-of-life care. Sept 2005. Cited Mar 5, 2006. Available from http://www.caot.ca/pdfs/National $\% 20$ Strategy $\% 20$ Final $\%$ 20Draft.pdf.

2. Hospice and Palliative Care Manitoba. Fact Sheet Hospice Palliative Care in Canada. 2004. Cited Feb 10, 2006. Available from http://www. manitobahospice.mb.ca/Factsheet_HospicePalliativeCareinCanada_ Feb2004_EN.pdf.

3. Singer P, Bowman KW. Quality end-of-life care: A global perspective. BMC Palliat Care. 2002;1:4.

4. Miaskowski C, Dodd M, Lee K. Symptom clusters: the new frontier in symptom management research. $J$ Natl Cancer Inst Monogr. 2004;32:17-21.

5. Dodd MJ, Miaskowski C, Paul SM. Symptom clusters and their effect on the functional status of patients with cancer. Oncol Nurs Forum. 2001;28:465-470.

6. Tokunaga E, Oki E, Nishida K, et al. Trastuzumab and breast cancer: developments and current status. Int J Clin Oncol. 2006;11:199-208.

7. DelVecchio Good MJ, Gadmer NM, et al. Narrative nuances on good and bad deaths: internists' tales from high-technology work places. Soc Sci Med. 2004;58:939-953.

8. Gysels M, Richardson A, Higginson IJ. Communication training for health professionals who care for patients with cancer: a systematic review of training methods. Support Care Cancer. 2005;13:356-366. 
